\title{
Design of a New Molecularly Imprinted Polymer Selective for Hydrochlorothiazide Based on Theoretical Predictions Using Gibbs Free Energy
}

\author{
Leonardo A. Barros, ${ }^{a}$ Rogério Custodio ${ }^{b}$ and Susanne Rath ${ }^{* a}$ \\ ${ }^{a}$ Departamento de Química Analítica and ${ }^{b}$ Departamento de Físico-Química, Instituto de Química, \\ Universidade Estadual de Campinas, P.O. Box 6154, 13084-971 Campinas-SP, Brazil
}

\begin{abstract}
A molecularly imprinted polymer (MIP) for the diuretic drug hydrochlorothiazide (HCTZ) based on theoretical predictions was developed. Molecular modeling calculations were performed to study the intermolecular interactions in the pre-polymerization mixture and to select a suitable functional monomer and a porogenic solvent for the synthesis of the MIP. To confirm the results of the theoretical predictions, three MIPs were synthesized and evaluated using the equilibrium batch rebinding method. A water-compatible MIP was prepared using HCTZ as the template and acrylamide as the functional monomer (FM) with ethylene glycol dimethacrylate as the cross-linker and tetrahydrofuran as the porogen. An imprinting factor of 8.24 was obtained. The polymer was characterized by Fourier transform infrared (FTIR), solid-state nuclear magnetic resonance, scanning electron microscopy, thermogravimetric analysis and nitrogen sorption porosimetry. In addition to HCTZ, six structurally related compounds were tested to evaluate the selectivity of the HCTZ-MIP, and cross-selectivity of the MIP was verified.
\end{abstract}

Keywords: molecularly imprinted polymer, molecular modeling, Gibbs free energy, hydrochlorothiazide, polarizable continuum model

\section{Introduction}

Molecular imprinting is a technique that creates recognition sites that are specific for a target molecule, called a template, within a synthetic polymer, and it has been widely used for the selective adsorption of drugs and their metabolites for analytical purposes. ${ }^{1-4}$ Comparable to immunosorbents, the specific binding sites are assigned to the specific interactions between the template and the functional groups in the polymer network, acting similarly to an antigen-antibody system..$^{5-7}$

The synthesis of a MIP first involves the complexation in solution of a template with a functional monomer (FM) through non-covalent or covalent interactions, followed by the polymerization of these monomers around the template in the presence of a cross-linker, a radical initiator and an appropriate solvent. After polymerization, in which the set FM-template is "frozen" into a three-dimensionally crosslinked rigid structure, the template is extracted from the polymer network, leaving its imprint and the cavities with size, shape and chemical functionality complementary to the template in the polymer structure.

*e-mail: susanne.rath@gmail.com
The formation of a complex between the template and FM through non-covalent interactions, i.e., hydrogen bonding, van der Waals forces, $\pi-\pi$ and electrostatic interactions, is the first and crucial step in the preparation of MIPs. The FM that can interact most strongly with the template provides the most stable complex. ${ }^{8}$

In the past, the optimal conditions for the preparation of MIPs were conducted mainly by an empirical trial and error approach. However, it has been shown in the literature that theoretical methods are attractive tools for the rational establishment of the selection of the FM and porogen solvent. ${ }^{9-11}$ In this context, different methods have been recommended, ranging from quantum mechanical simulations to statistical treatments. The most appropriate criterion to evaluate the spontaneity of a process is described by the Gibbs free energy. Nonetheless, some authors have preferred to use the $a^{b}$ initio $^{12}$ or Hartree-Fock electronic energies. In a previous work, we used a novel computational approach based on the calculation of the Gibbs free energy to predict the optimal conditions to prepare a MIP for fenitrothion, the results of which were validated by an experimental approach. ${ }^{13}$

Density functional theory (DFT) calculations have previously been used to select the best FM and porogenic 
solvent among a set of reagents traditionally used in the formulation of a non-covalent MIP. ${ }^{12-17}$ Until recently, molecular modeling has been used predominantly to study the interaction of the template with the FM and its influence on molecular recognition by the MIP. Only few papers reported in the literature have considered the effect of the solvent. Reports have shown that methods such as the polarizable continuum model (PCM) provide the possibility of including solvent effects on energy calculations of complexes in pre-polymerization mixtures. ${ }^{12,13,18}$ This solvent effect is approximated by placing the system into a cavity with a surface that is polarizable in accordance with the dielectric constant of the patterned solvent. ${ }^{18}$

The aim of this study was to develop a MIP for the determination of hydrochlorothiazide (HCTZ), a thiazide diuretic widely employed as a first-line therapy for hypertension, either alone or in combination with other antihypertensive medications. Diuretics are also often abused by athletes to excrete water for rapid weight loss and to mask the presence of other banned substances. Therefore, they have been included on the World Anti-Doping Agency's (WADA) list of prohibited substances. ${ }^{19}$ For the molecular modeling, the DFT at the B3LYP/6-31G(d) level and the PCM were adopted, and the theoretical studies were validated experimentally. It is worth emphasizing that a MIP for the recognition of HCTZ has previously been reported $;{ }^{20}$ however, the authors based their optimization on an empirical trial and error procedure.

In the present work, a more rigorous and correct calculation of the conditions of spontaneity of the system were considered to design a MIP for HCTZ, and the theoretical prediction was validated experimentally. Although this type of evaluation is not new in theoretical chemistry, it has so far been unexplored for analytical chemists.

\section{Experimental}

\section{Materials and reagents}

Hydrochlorothiazide (HCTZ) (99\%), chlorothiazide (CLTZ) (99\%), hydroflumethiazide (HFTZ) (97\%), chlorthalidone (CTLD) (99\%), indapamide (IDAM) (99\%), benzothiazide (BZTZ) (99\%), bendroflumethiazide (BFTZ) (99\%), acrylamide (AAM) (99\%), 4-vinylpiridine (95\%) (4-VP), allylamine (ALLY) (98\%) methacrylic acid (MAA) (98\%), ethylene glycol dimethacrylate (EGDMA), trimethylolpropanetrimethacrylate (TRIM) and divinylbenzene (DVB) were purchased from Sigma-Aldrich (St. Louis, USA). Methacrylic acid (MAA) (98\%) was purchased from Fluka (Buchs, Switzerland), and 2,2'-azobis-iso-butyronitrile (AIBN) was obtained from Sigma-Aldrich (Munich, Germany). All of the reagents were used without further purification and were at least of analytical grade. High performance liquid chromatography (HPLC)-grade solvents acetonitrile (ACN) and methanol $(\mathrm{MeOH})$ were obtained from Tedia (Fairfield, USA), tetrahydrofuran (THF) was obtained from Synth (São Paulo, Brazil) and acetic acid was obtained from Nuclear (São Paulo, Brazil). Dimethyl sulfoxide (DMSO), acetic acid (HAc), dimethylformamide (DMFA) and formic acid (FA) were purchased from Synth (São Paulo, Brazil). Throughout the study, water was obtained from a Milli-Q system from Millipore (São Paulo, Brazil).

Safety: special precautions are required when ALLY and 4-VP are handled because they are toxic and may cause burns and sensitization by inhalation when in contact with skin.

\section{Molecular modeling studies}

Molecular modeling simulations were performed using Gaussian 09 software. ${ }^{21}$ All the structures were minimized to the lowest energy conformation in vacuum at the B3LYP/6-31G(d) level. To calculate the Gibbs free energy, the optimization of the geometries of the template molecule (HCTZ), FM and the FM-HCTZ complex were fully optimized. The energies (E) of the compounds in atomic units (Hartrees) were converted to kcal $\mathrm{mol}^{-1}$ by multiplying the value by a factor of $627.51 \mathrm{kcal} \mathrm{mol}^{-1}$ Hartrees $^{-1}$. From the optimized geometries the harmonic vibrational frequencies of each compound were calculated. The thermal energies as well as the electronic, vibrational, rotational and translational entropies were obtained at $298.15 \mathrm{~K}$.

The absolute internal energy $\left(\mathrm{U}_{\mathrm{T}}, \mathrm{kcal} \mathrm{mol}^{-1}\right)$ of each molecule (HCTZ and FM) and super molecule (the complex FM-HCTZ) were obtained by equation 1 :

$\mathrm{U}_{\mathrm{T}}=\left(\mathrm{E}_{\text {elect }}+\mathrm{E}_{\mathrm{vib}}+\mathrm{E}_{\text {rot }}+\mathrm{E}_{\text {transs }}\right) \times 627.51$

where $E_{\text {elect }}$ is the electronic ground state energy at the equilibrium geometry; $\mathrm{E}_{\mathrm{vib}}, \mathrm{E}_{\mathrm{rot}}$ and $\mathrm{E}_{\text {transl }}$ are the vibrational, rotational and translational thermal energies, respectively.

For the calculation of the reaction internal energy $\left(\Delta \mathrm{U}, \mathrm{kcal} \mathrm{mol}^{-1}\right.$ ) (equation 2), the HCTZ absolute internal energy $\left(\mathrm{U}_{\mathrm{T}_{\text {HCTZ }}}\right)$ and the FM absolute internal energy $\left(\mathrm{U}_{\mathrm{T}_{\mathrm{FM}}}\right)$ were subtracted from the complex absolute internal energy $\left(\mathrm{U}_{\mathrm{T}_{\mathrm{FM}-\mathrm{HCTZ}}}\right)$ :

$\Delta \mathrm{U}=\mathrm{U}_{\mathrm{T}_{\mathrm{FM}-\mathrm{HCTZ}}}-\mathrm{U}_{\mathrm{T}_{\mathrm{HCTZ}}}-\mathrm{U}_{\mathrm{T}_{\mathrm{FM}}}$ 
The value of the enthalpy $\left(\Delta \mathrm{H}, \mathrm{kcal} \mathrm{mol}^{-1}\right)$ was obtained through equation 3 :

$\Delta \mathrm{H}=\Delta \mathrm{U}+\Delta \mathrm{nRT}$

where $\Delta \mathrm{n}$ is the variation in the number of moles of the reaction, $\mathrm{R}$ is the universal gas constant and $\mathrm{T}$ is the temperature.

The total entropy $\left(\mathrm{S}_{\mathrm{T}}\right)$ of HCTZ, FM and FM-HCTZ, expressed in $\mathrm{cal} \mathrm{mol}^{-1} \mathrm{~K}^{-1}$, was determined by equation 4 :

$\mathrm{S}_{\mathrm{T}}=\mathrm{S}_{\text {elect }}+\mathrm{S}_{\mathrm{vib}}+\mathrm{S}_{\text {rot }}+\mathrm{S}_{\text {transl }}$

where $\mathrm{S}_{\text {elect }}, \mathrm{S}_{\mathrm{vib}}, \mathrm{S}_{\text {rot }}$ and $\mathrm{S}_{\text {transl }}$ are the electronic, vibrational, rotational and translational entropies, respectively.

The total variation in entropy $\left(\Delta \mathrm{S}\right.$, cal $\left.\mathrm{mol}^{-1} \mathrm{~K}^{-1}\right)$ (equation 5) was determined by subtracting the HCTZ total entropy $\left(\mathrm{S}_{\mathrm{T}_{\text {HстZ }}}\right)$ and FM total entropy $\left(\mathrm{S}_{\mathrm{T}_{\mathrm{FM}}}\right)$ from the complex total entropy $\left(\mathrm{S}_{\mathrm{T}_{\text {complex }}}\right)$ :

$\Delta \mathrm{S}=\mathrm{S}_{\mathrm{T}_{\text {complex }}}-\mathrm{S}_{\mathrm{T}_{\mathrm{HCTZ}}}-\mathrm{S}_{\mathrm{T}_{\mathrm{FM}}}$

Finally, $\Delta \mathrm{G}\left(\mathrm{kcal} \mathrm{mol}^{-1}\right)$ was obtained from equation 6 :

$\Delta \mathrm{G}=\Delta \mathrm{H}-\left(\frac{\mathrm{T} \Delta \mathrm{S}}{1000}\right)$

Additionally, the solvent effect for six different solvents (dimethyl sulfoxide, acetonitrile, methanol, acetone, dichloromethane and tetrahydrofuran) was evaluated using the polarizable continuum model (PCM). ${ }^{11,22}$ Then, the Gibbs free energy of solvation $\left(\Delta \mathrm{G}_{\text {solv }}\right)$ was estimated using equation 7 :

$\Delta \mathrm{G}_{\mathrm{solv}}=\Delta \mathrm{G}+\Delta \mathrm{E}_{\mathrm{solv}}$

where $\Delta \mathrm{E}_{\text {solv }}$ is the solvation energy of the compounds in each specific solvent.

Polymer synthesis

Five different MIPs were prepared by bulk polymerization according to the non-covalent approach. $\operatorname{HCTZ}(0.5 \mathrm{mmol})$ as the template, AAM, MAA, 4-VP or $\operatorname{ALLY~}(1,2,3$ or $4 \mathrm{mmol})$ as the FM, EGDMA, TRIM or DVB $(10 \mathrm{mmol})$ as the cross-linker and AIBN $(0.24 \mathrm{mmol})$ as the free radical initiator were dissolved in $5 \mathrm{~mL}$ solvent (THF or DMSO) in a $75 \mathrm{~mL}$ thick-walled glass tube (Table 1). The mixture was homogenized appropriately in an ultrasonic bath and purged with oxygen-free nitrogen for $15 \mathrm{~min}$. The glass tube was sealed with parafilm under nitrogen, and polymerization was conducted in a thermostated oil bath at $60{ }^{\circ} \mathrm{C}$ for $24 \mathrm{~h}$. The obtained monolithic polymer was then crushed, ground and sieved using a 250 mesh sieve. Next, the pressurized liquid extraction (PLE) technique was used to remove the template from the polymer and the remaining reagents of the synthesis. The extraction of approximately $1.0 \mathrm{~g}$ of the prepared HCTZ-MIP was performed in a 34-mL extraction cell with an ASE $350^{\circledR}$ Accelerated Solvent Extractor (Dionex Corporation, Sunnyvale, USA). The extraction conditions were as follow, solvent: MeOH:DMFA:HAc (5:4:1, v/v/v); temperature: $85^{\circ} \mathrm{C}$; warm-up: $5 \mathrm{~min}$; number of cycles: 6 ; static time: $8 \mathrm{~min}$ and purge time: $180 \mathrm{~s}$. Under these PLE conditions, HCTZ was no longer detected in the solution using a previously established high-performance liquid chromatography-photodiode array detector (HPLC-DAD) method. Finally, the polymer was extracted with $\mathrm{MeOH}$ to remove the residual acetic acid. The polymer was dried overnight at $60{ }^{\circ} \mathrm{C}$ and stored at room temperature in a desiccator over silica gel. Non-imprinted polymers were prepared under identical conditions, except that there was no template present during the polymerization process.

Table 1. Reagents used in the polymer synthesis

\begin{tabular}{lcc}
\hline Polymer & Functional monomer & Solvent \\
\hline MIP 1 & AAM & THF \\
NIP 1 & AAM & THF \\
MIP 2 & AAM & DMSO \\
NIP 2 & AAM & DMSO \\
MIP 3 & MAA & THF \\
NIP 3 & MAA & THF \\
MIP 4 & 4-VP & THF \\
NIP 4 & 4-VP & THF \\
MIP 5 & ALLY & THF \\
NIP 5 & ALLY & THF \\
\hline
\end{tabular}

AAM: Acrylamide; MAA: methacrylic acid; 4-VP: 4-vinylpyridine; ALLY: allylamine.

Polymer characterization

Fourier transform infrared (FTIR) spectra of the polymers were obtained in $1 \% \mathrm{KBr}$ pellets with an $\mathrm{ABB}$ Bomem MB Series (Quebec, Canada) spectrometer (model B100) between $4000-400 \mathrm{~cm}^{-1}$ with a resolution of $4 \mathrm{~cm}^{-1}$.

Cross-polarization/magic angle spinning nuclear magnetic resonance spectra $\left({ }^{13} \mathrm{C} \mathrm{CP}-\mathrm{MAS}\right)$ were recorded on a Bruker Avance II $400 \mathrm{MHz}$ from Bruker Bio Spin (Rheinstetten, Germany) at $300 \mathrm{MHz}$ with a contact time of $4 \mathrm{~ms}$, a repetition interval of $3 \mathrm{~s}$ and an acquisition signal time of $50 \mathrm{~ms}$. 
Particle size analyses were performed using a JEOL 6360 LV-JSM (Tokyo, Japan) instrument operating at $15 \mathrm{keV}$. The polymer particles were sputter-coated with gold up to a thickness of $12 \mathrm{~nm}$ using a Bal-Tec MED 020 (Balzers, Liechtenstein) coater prior to obtaining the scanning electron microscopy (SEM) measurements.

The pore size distribution and surface areas of the washed polymers were measured with a multipoint Brunauer-Emmet-Teller (BET) analysis performed using Quantachrome Analysis ${ }^{\circledR}$ equipment at cryogenic temperatures. The pore volume and average pore diameter were determined using the multipoint BJH (Barret, Joyner, Halenda) model on desorption. The adsorption and desorption isotherms were constructed to provide relevant information about the surface area, total pore volume and average pore diameter of the polymers.

Thermogravimetry (TG) and derivative thermogravimetry (DTG) measurements were performed with a 5100 Thermal Analysis Instrument (TA Instruments). Samples were heated from ambient temperature to $800{ }^{\circ} \mathrm{C}$ at a heating rate of $10^{\circ} \mathrm{C} \mathrm{min}^{-1}$ under nitrogen and oxygen flows $\left(50 \mathrm{~mL} \mathrm{~min}^{-1}\right)$.

\section{Chromatographic conditions}

The separation and quantitation of the analytes by HPLC-DAD (Figure S1) was performed using a Zorbax Plus Phenyl-Hexyl column $(150 \times 4.6 \mathrm{~mm}, 5 \mu \mathrm{m})$ from Agilent, with a guard column $(4.0 \times 4.6 \mathrm{~mm}, 5 \mu \mathrm{m})$ containing the same stationary phase. The mobile phase was a mixture of $\mathrm{ACN}$ and formic acid (FA), $\mathrm{pH} 3$ with the following gradient elution: 0 to $0.5 \mathrm{~min}, 7: 93 \mathrm{v} / \mathrm{v} \mathrm{ACN}: \mathrm{FA}$; 0.5 to $2.5 \mathrm{~min}, 19: 81 \mathrm{v} / \mathrm{v}$ ACN:FA; 7 to $9 \mathrm{~min}, 60: 40 \mathrm{v} / \mathrm{v}$ ACN:FA; 9 to $11 \mathrm{~min}, 50: 50 \mathrm{v} / \mathrm{v} \mathrm{ACN}: \mathrm{FA}$ and 13 to 14 min, initial condition ( $7: 93 \mathrm{v} / \mathrm{v}$ ACN:FA). The quantitations were performed by external calibration at the following wavelengths: $230 \mathrm{~nm}$ (CTLD), $240 \mathrm{~nm}$ (IDAM), $270 \mathrm{~nm}$ (HCTZ, HFTZ and BFTZ), $277 \mathrm{~nm}$ (CLTZ) and $281 \mathrm{~nm}$ (BZTZ). The flow rate was $1.0 \mathrm{~mL} \mathrm{~min}^{-1}$.

The standard solutions $\left(500 \mu \mathrm{g} \mathrm{mL}^{-1}\right)$ were prepared by dissolving the accurately weighed standards $( \pm 0.01 \mathrm{mg})$ of HCTZ, CLTZ, HFTZ, CTLD, IDAM, BZTZ and BFTZ in methanol. The solutions were stored in the dark at $4{ }^{\circ} \mathrm{C}$. The working solutions were prepared prior to use by appropriate dilution of the standard solutions with $50: 50 \mathrm{v} / \mathrm{v}$ $\mathrm{ACN}$ :formic acid, $\mathrm{pH}$ 3. Prior to HPLC analysis, the samples were filtered through a $0.22 \mu \mathrm{m}$ nylon filter from Millipore. The calibration graph was linear in the range of 1.0 to $12.5 \mu \mathrm{g} \mathrm{mL}-1$ for all analytes with a correlation coefficient $(r)>0.99$. The limit of detection of HCTZ was $0.05 \mu \mathrm{g} \mathrm{mL} \mathrm{m}^{-1}$.

\section{Equilibrium batch rebinding studies}

Batch rebinding studies were performed for different purposes, including to evaluate the molecular modeling predictions and to evaluate the imprinting factors, the selectivities of the imprinted polymers and the adsorption isotherms.

For these purposes, $30 \mathrm{mg}$ quantities of the dry polymer were transferred to $15 \mathrm{~mL}$ glass flasks, and $5 \mathrm{~mL}$ of a solution of HCTZ and other thiazide diuretics at $10 \mu \mathrm{g} \mathrm{mL}^{-1}$, prepared in ACN, was added. Then, the flasks were properly sealed, and the mixture was incubated under agitation in a horizontal shaker from Marconi (Piracicaba, Brazil), model MA139/ $\mathrm{CFT}$, for $24 \mathrm{~h}$. After the rebinding process was completed, the mixture was centrifuged $(20 \mathrm{~min}, 6400 \times \mathrm{g})$, and the concentration of the free analyte was determined by HPLCDAD. The amount of analyte bound to the polymer was calculated by subtracting the amount of free analyte from its initial concentration, and the distribution coefficient $\left(\mathrm{K}_{\mathrm{D}}\right)$ and the imprinting factor (IF) were calculated.

For the adsorption isotherms, $15 \mathrm{mg}$ of MIP or NIP was incubated under agitation for $24 \mathrm{~h}$ with $2.5 \mathrm{~mL}$ of the HCTZ solution in ACN at eight concentration levels that ranged from 1 to $500 \mu \mathrm{g} \mathrm{L} \mathrm{L}^{-1}$.

The data were analyzed using the computational software Origin 8 from OriginLab Corporation (Northampton, MA, USA).

\section{Selectivity of MIP}

In addition to HCTZ, six structurally related compounds, CLTZ, HFTZ, CTLD, IDAM, BZTZ and BFTZ, were employed to evaluate the selectivity of the imprinted polymer. A volume of $5 \mathrm{~mL}$ of an $\mathrm{ACN}$ solution containing HCTZ or the analogue compound $\left(10 \mu \mathrm{gL}^{-1}\right)$ was added to $30 \mathrm{mg}$ of MIP or NIP in a $15 \mathrm{~mL}$ glass flask. The flasks were sealed, and the mixtures were incubated under agitation in the horizontal shaker for $24 \mathrm{~h}$. Aliquots of the supernatant were collected, and the analytes (HCTZ or analogue) were quantified by HPLC-DAD. The amount of analyte that was bound to the polymers was calculated by subtracting the amount of free analyte from its initial concentration, and the distribution coefficient $\left(\mathrm{K}_{\mathrm{D}}\right)$ and the selectivity coefficients $(\alpha)$ were calculated.

\section{Results and Discussion}

Theoretical selection of the FM and the solvent

The first step in the synthesis of a MIP for HCTZ involves the formation of a pre-polymerization complex 
between the FM and the template molecule (HCTZ). The FM that is able to interact most strongly with the template will provide a complex with the greatest stability.

In order to predict the properties of the MIP at the molecular level, the conformations of HCTZ, the FM and FM-HCTZ complexes were optimized to the lowest energy level using the DFT method, the B3LYP/6-31G(d) level of theory. In this work, the following FMs were selected for theoretical evaluations: methacrylic acid (MAA), acrylic acid (AA), 2-(trifluoromethyl)acrylic acid (TFMAA), acrylamide (AAM), methacrylamide (MAAM), 4-vinylpyridine (4-VP) and allylamine (ALLY).

The structure of HCTZ (Figure 1) revealed three main sites of possible interactions for complexation with the FM: one at the thiazide ring and two with the sulfonamide functional group. These three possible binding sites were simultaneously considered in the calculations because the
FM-HCTZ complexes in the molar ratio of 1:3 HCTZ:FM generated the most thermodynamically and energetically favorable, i.e., stable, complexes. The molecular modeling calculations were performed in vacuum and in solvents with different dielectric constants. The evaluated solvents in terms of decreasing values of dielectric constant were: dimethyl sulfoxide (DMSO), acetonitrile (ACN), methanol $(\mathrm{MeOH})$, acetone, dichloromethane (DCM) and tetrahydrofuran (THF). Table 2 summarizes the calculated interaction energies for the 1:3 complexes in the gas phase and in the evaluated solvents.

It is worth emphasizing that theoretical calculations performed in the gas phase are not adequate to describe the behavior and characteristics of molecules in solution and systems, and their properties can change considerably depending on the environment in which they find themselves. Thus, the solvent effect was theoretically

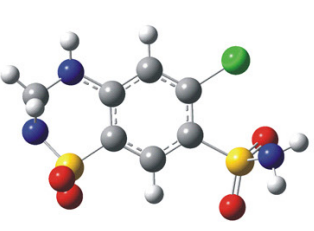

HCTZ

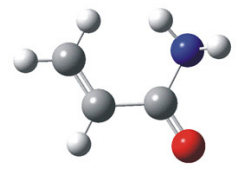

AAM
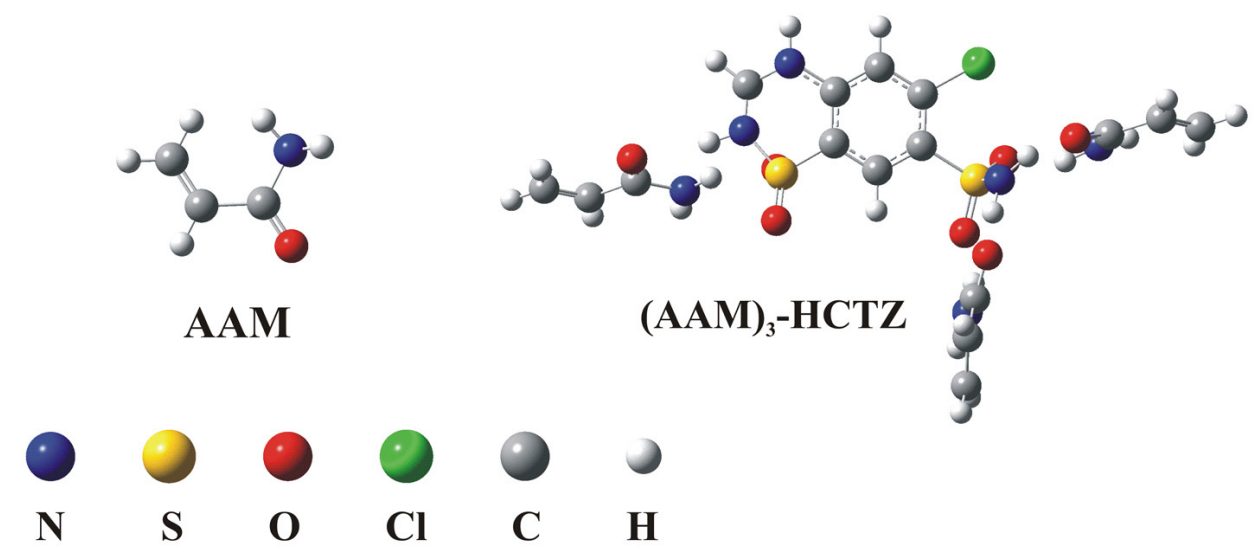

Figure 1. Optimized geometries of the most stable 1:3 (HCTZ:FM) complex of HCTZ with AAM.

Table 2. Gibbs energies of $\mathrm{HCTZ}-(\mathrm{FM})_{3}$ in vacuum and in different evaluated solvents

\begin{tabular}{|c|c|c|c|c|c|c|c|}
\hline \multirow[b]{2}{*}{ Complex } & \multicolumn{7}{|c|}{$\Delta \mathrm{G} /\left(\mathrm{kJ} \mathrm{mol}^{-1}\right)$} \\
\hline & Vacuum & $\begin{array}{c}\text { DMSO } \\
(\varepsilon=46.7)\end{array}$ & $\begin{array}{c}\mathrm{ACN} \\
(\varepsilon=37.5)\end{array}$ & $\begin{array}{c}\mathrm{MeOH} \\
(\varepsilon=33.0)\end{array}$ & $\begin{array}{l}\text { Acetone } \\
(\varepsilon=21.0)\end{array}$ & $\begin{array}{c}\text { DCM } \\
(\varepsilon=9.1)\end{array}$ & $\begin{array}{c}\text { THF } \\
(\varepsilon=7.5)\end{array}$ \\
\hline$(\mathrm{MAA})_{3}-\mathrm{HCTZ}$ & -2637.3 & -2503.5 & -2505.6 & -2493.4 & -2509.9 & -2523.7 & -2527.4 \\
\hline$(\mathrm{AA})_{3}-\mathrm{HCTZ}$ & -2795.8 & -2640.6 & -2661.5 & -2662.8 & -2669.3 & -2688.3 & -2695.0 \\
\hline$(\mathrm{TFMAA})_{3}-\mathrm{HCTZ}$ & -2774.7 & -2508.9 & -2525.2 & -2527.1 & -2529.4 & -2544.0 & -2547.8 \\
\hline$(\mathrm{AAM})_{3}-\mathrm{HCTZ}$ & -2637.3 & -2669.7 & -2707.3 & -2708.3 & -2712.0 & -2713.9 & -2727.8 \\
\hline$(\mathrm{MAAM})_{3}-\mathrm{HCTZ}$ & -2656.0 & -2548.5 & -2549.6 & -2550.6 & -2553.4 & -2564.1 & -2566.8 \\
\hline$(4-\mathrm{VP})_{3}-\mathrm{HCTZ}$ & -2575.4 & -2492.4 & -2494.2 & -2495.2 & -2496.3 & -2504.4 & -2506.4 \\
\hline$(\mathrm{ALLY})_{3}-\mathrm{HCTZ}$ & -2514.7 & -2449.3 & -2450.7 & -2451.8 & -2452.3 & -2459.2 & -2460.9 \\
\hline
\end{tabular}

MAA: Methacrylic acid; AA: acrylic acid; TFMAA: 2-(trifluoromethyl)acrylic acid; AAM: acrylamide; MAAM: methacrylamide; 4-VP: 4-vinylpyridine; ALLY: allylamine; $\varepsilon$ : dielectric constant. 
evaluated using the polarizable continuum model (PCM). The interaction energies (Gibbs energy) obtained from different solvents from the PCM corresponded in every case to a decrease compared to the gas phase interaction. This observation was consistent because the solvation of the different species also involved intermolecular interactions of the same nature as the monomer-template; therefore, the solvent acted as a competitor in the complex formation. ${ }^{12}$

The data verified that the Gibbs energy decreased (i.e., became more negative) as the solvent dielectric constant decreased (Table 2). Additionally, the data showed that the FM that resulted in the highest energy of interaction with the template was AAM, while the FM with the lowest energy of interaction was ALLY, regardless of the solvent characteristics. For the FM-template complex, a more negative Gibbs energy indicated a more thermodynamically favorable complexation process in which the solvent interfered less in the interaction between the template and the FM. THF was selected to prepare the MIP due to the favored solubility of HCTZ in this solvent. In relation to the $(\mathrm{FM})_{3}$-HCTZ complex, the stability order in THF was $\Delta \mathrm{G}_{\mathrm{AAM}}>\Delta \mathrm{G}_{\mathrm{AA}}>\Delta \mathrm{G}_{\mathrm{MAAM}}>\Delta \mathrm{G}_{\mathrm{TFMAA}}>\Delta \mathrm{G}_{\mathrm{MAA}}>$ $\Delta \mathrm{G}_{4-\mathrm{VP}}>\Delta \mathrm{G}_{\mathrm{ALLY}}$. To examine the predictive capability of the theoretical calculations, three polymers for HCTZ were prepared by bulk polymerization with the following porogenic solvents: AAM/THF (MIP 1), AAM/DMSO (MIP 2) and MAA/THF (MIP 3).

\section{Template: functional monomer ratio and cross-linker}

Many variables, such as the amount and nature of the FM, cross-linker and solvent, can affect the final characteristics of the materials obtained in terms of capacity, affinity and selectivity of the target analytes. Thus, non-specific interactions should be minimized by achieving an optimum combination of FM and cross-linker. According to the theoretical results of the molecular modeling, AAM and THF were selected as the FM and solvent, respectively.

Batch rebinding studies were performed to verify the adsorption of HCTZ to the different polymers synthesized in this work. After the adsorption process, the free HCTZ concentration was determined from previously constructed calibration curves in solvent. The distribution coefficients $\left(\mathrm{K}_{\mathrm{D}}\right)$ were calculated using equation 8 :

$\mathrm{K}_{\mathrm{D}}=\frac{\left(\mathrm{C}_{\mathrm{i}}-\mathrm{C}_{\mathrm{f}}\right) \times \mathrm{V}}{\mathrm{C}_{\mathrm{f}} \times \mathrm{m}}$

where $\mathrm{C}_{\mathrm{i}}, \mathrm{C}_{\mathrm{f}}, \mathrm{V}$ and $\mathrm{m}$ represent the drug concentration before adsorption $\left(\mathrm{mg} \mathrm{mL}^{-1}\right)$, the drug concentration after adsorption $\left(\mathrm{mg} \mathrm{mL}^{-1}\right)$, the volume of the solution $(\mathrm{mL})$ and the mass of the polymer, respectively. Finally, the imprinting factor (IF), a comparison of the coefficients obtained by analyzing the polymer synthesized with (imprinted) and without (non-imprinted) the template is defined in equation 9:

$\mathrm{IF}=\frac{\mathrm{K}_{\mathrm{D}}(\text { imprinted })}{\mathrm{K}_{\mathrm{D}}(\text { non-imprinted })}$

Typically, the appropriate molar ratios of FM to template are very important to improve the specific affinity of the polymers and the number of recognition sites of the MIP. High ratios of FM to template typically result in high non-specific affinity, while low ratios produce fewer complexation events due to insufficient functional groups. The cross-linker molar amount used in each of the formulations complied with the FM to cross-linker molar ratio of $1: 5,{ }^{6}$ and the amount of free radical initiator used was $2 \%$ of the total amount of monomer. Then, four molar ratios of the template to $\mathrm{FM}(1: 2,1: 4,1: 6$ and 1:8) were used and evaluated in the MIP preparations. The optimum ratio of template to FM for the specific rebinding of HCTZ was 1:4 (Table 3), which was demonstrated by the greater ability of the selective molecular recognition of the MIP relative to the NIP. The evaluated polymers were based on AAM, EGDMA and THF as the FM, cross-linker and porogenic solvent, respectively.

The function of the cross-linker in the polymer network is to arrange the functional groups of the FM into specific sites and directions around the template molecules and thereby maintain the binding site structure (specific cavities). The recognition capability of the MIP and their physical and chemical properties depend strongly on the degree of cross-linking and on the crosslinker nature. Three cross-linkers widely used in the synthesis of MIPs are EGDMA, TRIM and DVB, and these compounds were evaluated in this work. The molar ratio of template:FM:cross-linker used in this work was 1:4:20. Although some systems require a more resistant cross-linker such as DVB, EGDMA is used more often due to its ability to form thermally and mechanically stable polymers and its ability to enable rapid mass transfer during synthesis. This cross-linker combines a reactive methacrylate ester with a short spacer, allowing a large number of conformations and a certain degree of stiffness in the resulting polymer. ${ }^{25}$ Despite the bifunctional crosslinkers that predominate in most MIPs, other tri- and tetrafunctional monomers, such as pentaerythritoltriacrylate and TRIM may also be suitable. It was possible to verify (Table 4) that the MIP synthesized with EGDMA presented 
Table 3. Polymer compositions and calculated distribution coefficients $\left(\mathrm{K}_{\mathrm{D}}\right)$ and imprinting factors (IF)

\begin{tabular}{|c|c|c|c|c|c|c|c|}
\hline Polymer & $\begin{array}{c}\text { HCTZ / } \\
\text { mmol }\end{array}$ & $\begin{array}{l}\mathrm{AAM} / \\
\mathrm{mmol}\end{array}$ & $\begin{array}{c}\text { EGDMA / } \\
\text { mmol }\end{array}$ & $\begin{array}{c}\text { AIBN / } \\
\text { mmol }\end{array}$ & $\begin{array}{c}\mathrm{THF} / \\
\mathrm{mL}\end{array}$ & $\begin{array}{c}\mathrm{K}_{\mathrm{D}} / \\
\left(\mathrm{mL} \mathrm{g}^{-1}\right)\end{array}$ & IF \\
\hline MIP 1 & 0.5 & 1.0 & 10.0 & 0.24 & 5.0 & 55.6 & 1.56 \\
\hline NIP 1 & 0 & 1.0 & 10.0 & 0.24 & 5.0 & 35.7 & \\
\hline MIP 2 & 0.5 & 2.0 & 10.0 & 0.24 & 5.0 & 242.6 & 8.04 \\
\hline NIP 2 & 0 & 2.0 & 10.0 & 0.24 & 5.0 & 30.2 & \\
\hline MIP 3 & 0.5 & 3.0 & 10.0 & 0.24 & 5.0 & 189.9 & 2.80 \\
\hline NIP 3 & 0 & 3.0 & 10.0 & 0.24 & 5.0 & 67.9 & \\
\hline MIP 4 & 0.5 & 4.0 & 10.0 & 0.24 & 5.0 & 185.6 & 1.86 \\
\hline NIP 4 & 0 & 4.0 & 10.0 & 0.24 & 5.0 & 100.0 & \\
\hline
\end{tabular}

HCTZ: Hydrochlorothiazide; AAM: acrylamide; EGDMA: ethylene glycol dimethacrylate; AIBN: 2,2'-azobis-iso-butyronitrile; $\mathrm{K}_{\mathrm{D}}$ : distribution coefficient; IF: imprinting factor.

a higher IF than those synthesized with DVB or TRIM, and therefore the MIP synthesized with EGDMA possessed a greater capacity for selective recognition. Therefore, the results suggested that EGDMA was the best cross-linker for the synthesis of a HCTZ-based MIP.

\section{Polymer synthesis}

The imprinted polymer was synthesized through noncovalent interactions using HCTZ as the template, AAM as the FM, EGDMA as the cross-linker, AIBN as the radical initiator and THF as the porogenic solvent. With this approach, the complex between the FM and template was established by interactions such as hydrogen bonds, ionic bonds, van der Waals forces and/or hydrophobic effect forces. With this synthesis, the complex formed between the template and FM was established mainly by hydrogen bonds. Because the non-complexed monomers are randomly incorporated into the polymer matrix, resulting in non-imprinted or non-selective binding sites, a non-imprinted polymer was synthesized by the same procedure as the MIP synthesis, except for the addition of the template during the synthesis.

There are three possible interaction sites, one interaction site at the thiazide ring and two interaction sites at the sulfonamide functional group in the base structure of the diuretic drug. These groups can form hydrogen bonds with functional groups such as hydroxyl, carboxyl, amine and amide groups. The AAM was the best FM, which was indicated by molecular modeling studies (Theoretical selection of the FM and the solvent section), regardless of the characteristics of the porogenic solvent; therefore, the polymer preparation with the AAM-based polymeric backbone likely afforded improved efficiencies in the selective adsorption of the target analyte (template) in question, as noted by Song et al. ${ }^{27}$ Then, after AAM

Table 4. Evaluation of the cross-linker used during MIP synthesis

\begin{tabular}{|c|c|c|c|c|c|c|}
\hline Polymer & $\begin{array}{l}\mathrm{AAM} / \\
\mathrm{mmol}\end{array}$ & $\begin{array}{c}\text { Cross-linker / } \\
\text { mmol }\end{array}$ & $\begin{array}{c}\mathrm{AIBN} / \\
\mathrm{mL}\end{array}$ & $\begin{array}{c}\mathrm{THF} / \\
\mathrm{mL}\end{array}$ & $\begin{array}{c}\mathrm{K}_{\mathrm{D}} / \\
\left(\mathrm{mL} \mathrm{g}^{-1}\right)\end{array}$ & IF \\
\hline MIP 1 & 2.0 & $\begin{array}{c}\text { EGDMA } \\
20.0\end{array}$ & 0.24 & 5.0 & 224.0 & 7.34 \\
\hline NIP 1 & 2.0 & $\begin{array}{c}\text { EGDMA } \\
20.0\end{array}$ & 0.24 & 5.0 & 30.5 & \\
\hline MIP 2 & 2.0 & $\begin{array}{c}\text { TRIM } \\
20.0\end{array}$ & 0.24 & 5.0 & 306.7 & 3.11 \\
\hline NIP 2 & 2.0 & $\begin{array}{c}\text { TRIM } \\
20.0\end{array}$ & 0.24 & 5.0 & 98.6 & \\
\hline MIP 3 & 2.0 & $\begin{array}{l}\text { DVB } \\
20.0\end{array}$ & 0.24 & 5.0 & 148.1 & 1.78 \\
\hline NIP 3 & 2.0 & $\begin{array}{l}\text { DVB } \\
20.0\end{array}$ & 0.24 & 5.0 & 83.2 & \\
\hline
\end{tabular}

AAM: Acrylamide; EGDMA: ethylene glycol dimethacrylate; AIBN: 2,2'-azobis-iso-butyronitrile; THF: tetrahydrofuran; $\mathrm{K}_{\mathrm{D}}$ : distribution coefficient; IF: imprinting factor. 
polymerization and cross-linking by EGDMA, this spatial structure was "frozen". Additionally, AIBN was used as a radical initiator because its half-life at $60{ }^{\circ} \mathrm{C}$ is approximately 10 hours, ${ }^{27}$ and it therefore was suitable for the polymerization time selected in our studies.

After polymerization, the template must be removed. This procedure must be optimized to extract the template in a short time and to use low solvent volumes, in addition to minimizing the disruption of the selective sites of the MIP.

Typically, after polymerization, the template removal is achieved by extensive washing with "competitor" solvents such as MeOH/HAc (9:1, v/v) in a Soxhlet apparatus. This approach typically results in the removal of up to $99 \%$ of the template. ${ }^{28}$ In the present work, the template removal was performed using the PLE technique, which favored the complete removal (concentration less than the detection limit of the HPLC-DAD method) of the template from the polymer with a lower solvent consumption and less time spent on the extraction.

After the MIPs were synthesized, their specificity, binding capacity and selectivity for the target analyte were evaluated through adsorption (rebinding) studies in equilibrium. These studies are usually performed with the same solvent that was used for the polymer synthesis. Finally, an IF value was established between the MIP and the NIP. An ideal MIP is one whose control polymer rebinds little or none of the target analyte, consequently resulting in a high IF value. The selectivity for other compounds was also evaluated to check possible cross-selectivity. The evaluation of these compounds with the control polymer was also required because better or worse adsorption of a particular compound over another could simply be due to its specific physico-chemical properties and not to the imprinted specific sites.

Experimental validation of theoretical predictions: functional monomer and solvent

To examine the accuracy of the theoretical calculations, several HCTZ-based polymers were prepared by bulk polymerization using different FM and solvent combinations. The FMs selected to be evaluated experimentally were AAM, MAA, 4-VP and ALLY. Due to limitations related to the solubility of HCTZ, the selected solvents to be evaluated experimentally were THF and DMSO. When the FM 4-VP and ALLY were employed (MIP 4 and MIP 5, respectively), in THF, the formation of the pre-polymerization complex was not observed, and thus the formation of the polymer matrix was not achieved. This observation could be explained by the fact that these two monomers did not provide thermodynamically stable complexes with the template, which corroborated the results obtained from the theoretical calculations.

Table 5 shows the distribution coefficients of the HCTZ-imprinted polymers that were synthesized with AAM in THF (MIP 1), AAM in DMSO (MIP 2) and MAA in THF (MIP 3). The distribution coefficients for the nonimprinted polymers are also presented.

The highest $K_{D}$ and IF values were obtained for MIP 1 , which corroborated the theoretical predictions that AAM and THF allowed more stable and thermodynamically favorable interactions with HCTZ and consequently resulted in greater specificity of the selective sites during the molecular recognition process. Moreover, as expected by the results obtained from the theoretical studies, the synthesized polymer in THF yielded values of $K_{D}$ and IF that were greater than those synthesized in DMSO because THF has a lower dielectric constant relative to DMSO.

According to Kyzas et al. ${ }^{29}$ MIPs generally exhibit better performances in hydrophobic organic solvents such as chloroform or toluene. This observation can be explained by the fact that non-polar solvents eliminate non-specific hydrophobic interactions, and they create a better environment for electrostatic interactions that play an important role in molecular recognition.

\section{Equilibrium batch rebinding studies and selectivity of MIP}

Imprinted polymers generally display better performance with regard to molecular recognition when solvents with similar properties as used in the synthesis are employed. Rebinding studies for the target analyte (HCTZ) and for six structurally related compounds, CLTZ, HFTZ, CTLD, IDAM, BZTZ and BFTZ, shown in Figure 2, were performed in ACN. Despite the fact that the solvent used during synthesis was THF, the rebinding studies were performed in ACN because THF has higher eluotropic

Table 5. Distribution coefficients determined for the imprinted and non-imprinted polymers

\begin{tabular}{|c|c|c|c|c|c|c|}
\hline Polymer & MIP 1 & NIP 1 & MIP 2 & NIP 2 & MIP 3 & NIP 3 \\
\hline $\mathrm{K}_{\mathrm{D}} /\left(\mathrm{mL} \mathrm{g}^{-1}\right)$ & 217.2 & 27.0 & 123.8 & 45.0 & 37.8 & 28.1 \\
\hline IF & \multicolumn{2}{|c|}{8.0} & \multicolumn{2}{|c|}{2.8} & \multicolumn{2}{|c|}{1.3} \\
\hline
\end{tabular}

MIP 1: AAM in THF; MIP 2: AAM in DMSO and MIP 3: MAA in THF. 
strength than does ACN and therefore diminishes the adsorption of the analyte by the polymers. The calculated $K_{D}$, IF and selectivity coefficients $(\alpha)$ are shown in Table 6 , where the $\alpha$ value was calculated as following:

$\alpha=\frac{\mathrm{IF}(\text { template })}{\mathrm{IF}(\text { analogue })}$

The data shown in Table 6 indicate that both the MIP and NIP possessed some affinity for the structurally related compounds to varying extents.
HCTZ presented the highest $\mathrm{K}_{\mathrm{D}}$ and IF values, which was justified because the MIP was prepared using this compound as the template molecule. The second highest $\mathrm{K}_{\mathrm{D}}$ value was determined for the analogue HFTZ, an analogue of HCTZ in which the chlorine atom at the C6 of the benzothiadiazine ring is replaced by a $\mathrm{CF}_{3}$ group. However, the IF was lower by a factor of four compared to the HCTZ, indicating that this molecule rebinds mainly through non-specific interactions. The presence of nonspecific sites is justified due to the excess FM employed during the polymer preparation. CLTZ, which differs in<smiles>NS(=O)(=O)c1cc2c(cc1Cl)NCNS2(=O)=O</smiles>

HCTZ<smiles>NS(=O)(=O)c1cc2c(cc1C(F)(F)F)NCNS2(=O)=O</smiles>

HFTZ<smiles>NS(=O)(=O)c1cc2c(cc1Cl)N=CNS2(=O)=O</smiles>

CLTZ<smiles>NS(=O)(=O)c1cc(C2(O)NC(=O)c3ccccc32)ccc1Cl</smiles><smiles>CC1Cc2ccccc2N1NC(=O)c1ccc(Cl)c(S(N)(=O)=O)c1</smiles>

IDAM<smiles>NS(=O)(=O)c1cc2c(cc1C(F)(F)F)NC(Cc1ccccc1)NS2(=O)=O</smiles>

BFTZ<smiles>NS(=O)(=O)c1cc2c(cc1Cl)NC(CSCc1ccccc1)=NS2(=O)=O</smiles>

BZTZ

Figure 2. Structures of thiazide diuretics evaluated in the batch rebinding studies. HCTZ: hydrochlorothiazide, CLTZ: chlorothiazide, HFTZ: hydroflumethiazide, CTLD: chlorthalidone, IDAM: indapamide, BZTZ: benzothiazide; BFTZ: bendroflumethiazide.

Table 6. Distribution coefficients $\left(K_{D}\right)$, imprinting factors (IF) and selectivity coefficients $(\alpha)$ for the rebinding of different compounds to the molecularly imprinted and non-imprinted polymers synthesized using AAM and THF as the FM and solvent, respectively, and using HCTZ as the template

\begin{tabular}{|c|c|c|c|c|c|c|c|}
\hline Polymer & HCTZ & CLTZ & HFTZ & CTLD & IDAM & BZTZ & BFTZ \\
\hline $\begin{array}{l}\text { MIP } \\
\mathrm{K}_{\mathrm{D}} /\left(\mathrm{mL} \mathrm{g}^{-1}\right)\end{array}$ & 217 & 88 & 106 & 53 & 49 & 40 & 52 \\
\hline $\begin{array}{l}\text { NIP } \\
\mathrm{K}_{\mathrm{D}} /\left(\mathrm{mL} \mathrm{g}^{-1}\right)\end{array}$ & 27 & 36 & 36 & 39 & 40 & 39 & 36 \\
\hline IF & 8.04 & 2.45 & 2.97 & 1.38 & 1.23 & 1.02 & 1.42 \\
\hline$\alpha$ & - & 3.3 & 2.7 & 5.8 & 6.6 & 7.9 & 5.7 \\
\hline
\end{tabular}

HCTZ: Hydrochlorothiazide; CLTZ: chlorothiazide; HFTZ: hydroflumethiazide; CTLD: chlorthalidone; IDAM: indapamide; BZTZ: benzothiazide; BFTZ: bendroflumethiazide. 
structure from HCTZ by the presence of a double bond in the 3,4 position of the benzothiadiazine ring, presented the third highest $\mathrm{K}_{\mathrm{D}}$ value. The IF and $\alpha$ values of CLTZ were close to the values obtained for HFTZ. The other 4 analogues possessed significant structural differences in relation to HCTZ, which explained the lower $\mathrm{K}_{\mathrm{D}}$ values in the range of 43 to $53 \mathrm{~mL} \mathrm{~g}^{-1}$.

After evaluating the recognition of all the analogues with the MIP and NIP, it was possible to conclude that the synthesized MIP presented cross-selectivity for this class of compounds.

\section{Polymer characterization}

FTIR spectra of HCTZ-MIP, NIP and AAM are shown in Figure S2. The spectra of both the MIP and NIP presented the same characteristic absorption bands, which indicated that the template extraction procedure adopted using PLE was efficient enough to remove the HCTZ from the polymer and that the imprinted polymer did not incorporate the template molecule into the polymeric structure. Moreover, the $\mathrm{S}=\mathrm{O}$ bond of the sulfonamide group of HCTZ, which exhibits intense absorption between 1370 and $1335 \mathrm{~cm}^{-1}$ due to the axial deformation of the $\mathrm{S}=\mathrm{O}$ group, was not observed in the spectra. The main absorption bands included the following: $3500 \mathrm{~cm}^{-1}(\mathrm{OH}$ stretch in the $\mathrm{COOH}$ group of the monomer), $3001 \mathrm{~cm}^{-1}$ (OH stretch in the $\mathrm{COOH}$ dimer and axial deformation of $\left.\mathrm{CH}_{3}\right), 1733 \mathrm{~cm}^{-1}(\mathrm{C}=\mathrm{O}$ stretch of the ester), and $1267 \mathrm{~cm}^{-1}$ (angular deformation of the $\mathrm{C}-\mathrm{O}$ of the ester group). By analyzing the AAM spectrum and comparing the MIP and NIP spectra, it was possible to verify that the absorption bands at 3352 and 3198 (NH group axial deformation), 1679 ( $\mathrm{C}=\mathrm{O}$ group axial deformation) and $1617 \mathrm{~cm}^{-1}$ (NH group angular deformation) were no longer present, indicating that the AAM monomer was incorporated into the polymeric matrix.

The ${ }^{13} \mathrm{C}$ CP-MAS NMR spectra (Figure S3) also confirmed that both polymeric solids (MIP and NIP) exhibited the same carbon structure. The predominant resonances shown were attributed to the following: 20-80 ppm (methyl and methylene groups of EGDMA and AAM), 100-130 ppm (unreacted double bonds of the EGDMA) and 160-180 ppm (C=O of EGDMA). ${ }^{23}$

Micrographs of the MIP and the corresponding NIP polymers prepared with the same FM (Figure S4) revealed that they were similar in their morphologies. The morphologies of these polymers were in agreement with that of bulk polymerization, which leads to surfaces with irregular areas.

The porosities of the polymers synthesized with AAM in THF were obtained through nitrogen sorption porosimetry. Whereas the surface areas were obtained by the BET method, the volumes and average pore sizes were calculated by the BJH method. The results are shown in Table 7. The imprinted polymer presented a larger surface area than the non-imprinted polymer according to results found by Farrington and Reagan. ${ }^{9}$ Because both polymers were synthesized with the same volume of the same porogen solvent, they had similar average pore diameter values. The presence of the template molecule upon synthesis of the imprinted polymer led to an increased pore volume compared to the NIP, which was synthesized in the absence of the template.

Table 7. Surface areas, pore volumes and pore diameters for MIP and NIP synthesized with AAM as the FM and THF as the porogen solvent

\begin{tabular}{lccc}
\hline Polymer & $\begin{array}{c}\text { Surface area / } \\
\left(\mathrm{m}^{2} \mathrm{~g}^{-1}\right)\end{array}$ & $\begin{array}{c}\text { Pore volume / } \\
\left(\mathrm{cm}^{3} \mathrm{~g}^{-1}\right)\end{array}$ & $\begin{array}{c}\text { Average pore } \\
\text { diameter } / \mathrm{nm}\end{array}$ \\
\hline MIP & 340.5 & 0.44 & 3.87 \\
NIP & 164.2 & 0.27 & 3.79 \\
\hline
\end{tabular}

According to the experimentally obtained data, both polymers could be considered as mesoporous because they presented surface areas ranging from 10 to $500 \mathrm{~m}^{2} \mathrm{~g}^{-1}$ and possessed pores with average diameters that ranged from 2 to $50 \mathrm{~nm}$.

The thermal behavior of the HCTZ-MIP and NIP were analyzed by TG/DTG in oxygen and inert atmospheres. Mass losses of approximately 6 and $8 \%$ for the MIP and NIP materials, respectively, were observed in the temperature range of 25 to $100{ }^{\circ} \mathrm{C}$, which was related to the loss of water molecules that were weakly adsorbed onto the surfaces of the materials. The polymers began to decompose at $270{ }^{\circ} \mathrm{C}$. In the temperature range of 270-390 ${ }^{\circ} \mathrm{C}$, a 54 and $52 \%$ mass loss was measured for the MIP and NIP, respectively.

The TGA results showed that both the MIP and NIP were stable at the temperatures in which the template removal by PLE was realized $\left(85^{\circ} \mathrm{C}\right)$.

\section{Adsorption isotherms}

The selection of the appropriate adsorption model is primarily based on its ability to accurately reproduce the constructed isotherm from experimental data, and it should reflect the distribution of the bound sites in the measured system to generate real adsorption parameters. The Langmuir-Freundlich heterogeneous isotherm (LF) was considered in this work for the evaluation of the binding characteristics of the synthesized HCTZ-MIP. 
The LF isotherm describes a relationship between B and $\mathrm{F}$ (Figure 3) in heterogeneous systems with three different coefficients $\left(\mathrm{N}_{\mathrm{t}}, a\right.$ and $\left.m\right)$ according to equation 11 :

$\mathrm{B}=\frac{\mathrm{N}_{\mathrm{t}} a \mathrm{~F}^{m}}{1+a \mathrm{~F}^{m}}$

where $\mathrm{N}_{\mathrm{t}}$ is the total number of binding sites, $a$ is the average binding affinity and $m$ is the heterogeneous index, which is equal to 1 for a homogeneous material or ranges from 0 to 1 if the material is heterogeneous (Table 8).

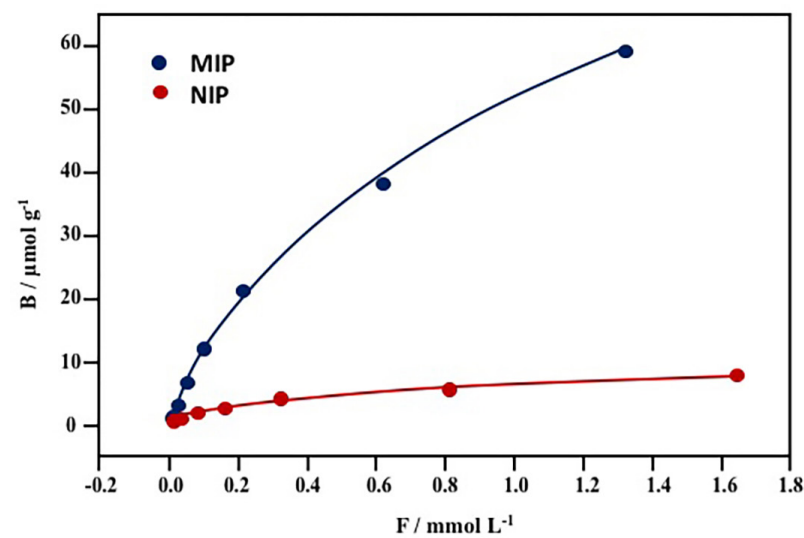

Figure 3. Adsorption isotherms of MIP and NIP using the LF model (B is the concentration of HCTZ adsorbed per gram of polymer, and F is the concentration of the free HCTZ).

Table 8. LF isotherm fitting parameters for MIP and NIP

\begin{tabular}{lcccc}
\hline Polymer & $\begin{array}{c}\mathrm{N}_{\mathrm{t}} / \\
\left(\mu \mathrm{mol} \mathrm{g}^{-1}\right)\end{array}$ & $\begin{array}{c}a / \\
\left(\mathrm{mmol}^{-1} \mathrm{~L}\right)\end{array}$ & $m$ & $\mathrm{R}^{2}$ \\
\hline MIP & $160 \pm 48$ & $0.48 \pm 0.21$ & 0.76 & 0.996 \\
NIP & $22 \pm 12$ & $0.41 \pm 0.22$ & 0.63 & 0.986 \\
\hline
\end{tabular}

$\mathrm{N}_{\mathrm{t}}$ : Total number of binding sites; $a$ : average binding affinity; $m$ : heterogeneous index; $\mathbf{R}^{2}$ : coefficient of determination.

The comparison of the binding parameters revealed that the HCTZ-MIP prepared with AAM in THF possessed a greater concentration of binding sites per gram of polymer $\left(\mathrm{N}_{\mathrm{t}}=160 \mu \mathrm{mol} \mathrm{g}^{-1}\right)$ and a higher average binding affinity $\left(a=0.48\left(\mathrm{mmol}^{-1} \mathrm{~L}\right)\right)$ than the NIP.

A plot of $\log \mathrm{B}$ vs. $\log \mathrm{F}$ must be linear for the experimental values to be described accurately by the LF isotherms, which was observed because the correlation coefficient value for the imprinted polymer was 0.993 .

\section{Behavior of the MIP in the extraction of HCTZ from synthetic} urine

The synthesized MIP and NIP were used in a solid phase extraction cartridge for the extraction of HCTZ from synthetic urine $\left(250 \mathrm{ng} \mathrm{mL}^{-1}\right)$ in order to evaluate the behavior of this polymers in aqueous solutions. For this purpose, $60 \mathrm{mg}$ of the MIP or NIP were introduced in an empty cartridge between two polyethylene frits. The sorbent was conditioned with $3 \mathrm{~mL}$ of THF, $3 \mathrm{~mL}$ of water and $3 \mathrm{~mL}$ of ammonium phosphate, $\mathrm{pH}$. The $\mathrm{pH}$ of the urine sample before loading was adjusted to 6.0. For the clean-up $1 \mathrm{~mL}$ of water was percolated through the cartridge and the elution was performed with three portions of $0.5 \mathrm{~mL}$ of a mixture of methanol:DMF:acetic acid 5:4:1 $\mathrm{v} / \mathrm{v} / \mathrm{v}$. The recovery of HCTZ from the MIP and NIP were 95.3 and $9.3 \%$, respectively. These results indicate that the proposed MIP is selective to HCTZ and has potential to be applied in the determination of HCTZ in urine, as well as in aqueous solutions.

\section{Conclusions}

The selection of the best imprinting conditions, such as the FM and porogenic solvent, is still commonly performed using an empirically based "trial and error" approach, which is tedious and time consuming. Our results showed that molecular modeling is a rational and rapid theoretical approach for the selection of appropriate conditions with regard to the FM and the porogenic solvent for successful molecular imprinting. The DFT and PCM calculations predicted that acrylamide and tetrahydrofuran would be the best combination of FM and solvent, respectively, for the synthesis of a MIP for HCTZ recognition. The experimental results obtained confirmed the data predicted by molecular modeling. The equilibrium batch rebinding studies showed that the adsorption of HCTZ to the polymer matrix fitted well to a Langmuir-Freundlich model. In addition, the polymeric material obtained was heterogeneous, and the MIP presented a higher number of binding sites than the NIP. Additionally, the synthesized HCTZ-MIP showed cross-selectivity for structural analogues such as CLTZ, HFTZ, CTLD, IDAM, BZTZ and BFTZ, which could be useful for analytical applications in which compounds of the same class must be determined. The imprinted polymer was used for the solid phase extraction of HCTZ in a synthetic urine sample and showed selectivity and adequate retention to be used in aqueous solutions.

\section{Supplementary Information}

Supplementary data (characteristic chromatogram, FTIR spectra, ${ }^{13} \mathrm{C}$ CP-MAS NMR spectra and scanning electron micrographs of MIP and NIP) are available free of charge at http://jbcs.sbq.org.br. 


\section{Acknowledgments}

The authors gratefully acknowledge financial support from CNPq (141618/2010-8), FAPESP (2007/02306-9) and FAEPEX-UNICAMP.

\section{References}

1. Chianella, I.; Karim, K.; Piletska, E. V.; Preston, C.; Piletsky, S. A.; Anal. Chim. Acta 2006, 559, 73-78.

2. Claude, B.; Morin, P.; Bayoudh, S.; de Ceaurriz, J.; J. Chromatogr. A 2008, 1196, 81.

3. Figueiredo, E. C.; Sparrapan, R.; Sanvido, G. B.; Santos, M. G.; Arruda, M. A. Z.; Eberlin, M. N.; Analyst 2011, 136, 3753.

4. Hoshina, K.; Horiyama, S.; Matsunaga, H.; Haginaka, J.; J. Pharm. Biomed. Anal. 2011, 55, 916.

5. Cormack, P. A. G.; Elorza, A. Z.; J. Chromatogr. B 2004, 804, 173.

6. Spivak, D. A.; Adv. Drug Deliver. Rev. 2005, 57, 1779.

7. Yan, M.; Ramström, O.; Molecularly Imprinted Materials: Science and Technology; Marcel Dekker: New York, 2005.

8. Dong, W. G.; Yan, M.; Zhang, M. L.; Liu, Z.; Li, Y. M.; Anal. Chim. Acta 2005, 542, 186.

9. Farrington, K.; Regan, F.; Biosens. Bioelectron. 2007, 22, 1138.

10. Liu, Y.; Wang, F.; Tan, T. W.; Lei, M.; Anal. Chim. Acta 2007, 581,137

11. Yao, J. H.; Li, X.; Qin, W.; Anal. Chim. Acta 2008, 610, 282.

12. Gholivand, M. B.; Khodadadian, M.; Ahmadi, F.; Anal. Chim. Acta 2010, 658, 225.

13. Barros, L. A.; Pereira, L. A.; Custódio, R.; Rath, S.; J Braz. Chem. Soc. 2014, 25, 619.

14. Dineiro, Y.; Menendez, M. I.; Blanco-Lopez, M. C.; LoboCastanon, M. J.; Miranda-Ordieres, A. J.; Tunon-Blanco, P.; Anal. Chem. 2005, 77, 6741.

15. Dineiro, Y.; Menendez, M. I.; Blanco-Lopez, M. C.; LoboCastanon, M. J.; Miranda-Ordieres, A. J.; Tunon-Blanco, P.; Biosens. Bioelectron. 2006, 22, 364.

16. Khodadadian, M.; Ahmadi, F.; Talanta 2010, 81, 1446.

17. Saloni, J.; Lipkowski, P.; Dasary, S. S. R.; Anjaneyulu, Y.; Yu, H. T.; Hill, G.; Polymer 2011, 52, 1206.
18. Nicholls, I. A.; Andersson, H. S.; Charlton, C.; Henschel, H.; Karlsson, B. C. G.; Karlsson, J. G.; O’Mahony, J.; Rosengren, A. M.; Rosengren, K. J.; Wikman, S.; Biosens. Bioelectron. 2009, 25, 543.

19. Cadwallader, A. B.; de la Torre, X.; Tieri, A.; Botre, F.; Brit. J. Pharmacol. 2010, 161, 1.

20. Rezaei, B.; Mallakpour, S.; Rahmanian, O.; J. Iran Chem. Soc. 2010, 7, 1004.

21. Frisch, M. J.; Trucks, G. W.; Schlegel, H. B.; Scuseria, G. E.; Robb, M. A.; Cheeseman, J. R.; Zakrzewski, V. G.; Montgomery, J. J. A.; Stratmann, R. E.; Burant, J. C.; Dapprich, S.; Millam, J. M.; Daniels, A. D.; Kudin, K. N.; Strain, M. C.; Farkas, O.; Tomasi, J.; Barone, V.; Cossi, M.; Cammi, R.; Mennucci, B.; Pomelli, C.; Adamo, C.; Clifford, S.; Ochterski, J.; Petersson, G. A.; Ayala, P. Y.; Cui, Q.; Morokuma, K.; Malick, D. K.; Rabuck, A. D.; Raghavachari, K.; Foresman, J. B.; Cioslowski, J.; Ortiz, J. V.; Stefanov, B. B.; Liu, G.; Liashenko, A.; Piskorz, P.; Komaromi, I.; Gomperts, R.; Martin, R. L.; Fox, D. J.; Keith, T.; Al-Laham, M. A.; Peng, C. Y.; Nanayakkara, A.; Gonzalez, C.; Challacombe, M.; Gill, P. M. W.; Johnson, B.; Chen, W.; Wong, M. W.; Andres, J. L.; Gonzalez, C.; Head-Gordon, M.; Replogle, E. S.; Pople, J. A.; GAUSSIAN 03, Revision C.01; Gaussian Inc: Pittsburgh, PA, 2003.

22. Wu, L. Q.; Zhu, K. C.; Zhao, W. P.; Li, Y. Z.; Anal. Chim. Acta 2005, 549, 39.

23. Celiz, M. D.; Aga, D. S.; Colon, L. A.; Microchem. J. 2009, 92, 174.

24. Muhammad, T.; Nur, Z.; Piletska, E. V.; Yimit, O.; Piletsky, S. A.; Analyst 2012, 137, 2623.

25. Sousa, M. D.; Barbosa, C. M.; Quim. Nova 2009, 32, 1609.

26. Kempe, M.; Mosbach, K.; Tetrahedron Lett. 1995, 36, 3563.

27. Song, X. L.; Li, J. H.; Wang, J. T.; Chen, L. X.; Talanta 2009 , $80,694$.

28. Bui, B. T. S.; Haupt, K.; Anal. Bioanal. Chem. 2010, 398, 2481.

29. Kyzas, G. Z.; Bikiaris, D. N.; Lazaridis, N. K.; Chem. Eng. J. 2009, 149, 263.

Submitted: January 19, 2016

Published online: April 26, 2016

FAPESP has sponsored the publication of this article. 